\title{
Application of Mini-UAV in Emergency Rescue of Major Accidents of Hazardous Chemicals
}

\author{
WANG Li-li \\ Jiangsu Academy of Safety Science \& Technology \\ Nanjing, China \\ lily.w_aky@yahoo.com.cn \\ ZHOU Wang-ping \\ School of Information \& Control Engineering, Nanjing \\ University of Information Science \& Technology
}

\author{
Nanjing, China \\ wpzhou@yahoo.cn \\ ZHAO Shi-lin \\ Jiangsu Academy of Safety Science \& Technology \\ Nanjing, China \\ 308255154@qq.com
}

\begin{abstract}
The major accidents of hazardous chemicals always occur suddenly and result in rapid proliferation, wide range of damage as well as grave consequences. So that quick, accurate and effective response to the situation after the accidents is the most crucial part of the rescue. Meanwhile, the timely information of the accidents is of great importance to the formulating of rescue strategy and furthermore, the improving of rescue efficiency and quality. Mini-UAV's (unmanned aerial vehicle) has certain characteristics like real-time, flexible, low cost, and most of all, it can be operated in high-risk areas. This paper introduces the composition of its aerial remote sensing detection system and the application of Mini-UAVs in emergency rescue of major accidents of hazardous chemicals, especially the application of the system in the emergent processing of oil spill. At last, this paper summarizes the advantages of Mini-UAV in emergency rescue of major accidents of hazardous chemicals and points out the deficiency of the system and the ways to improve it.
\end{abstract}

Index Terms-UVA. hazardous chemicals. Accidents. emergency rescue.

\section{INTRODUCTION}

The major accidents of hazardous chemicals always occur suddenly and result in rapid proliferation, wide range of damage as well as grave consequences so that the response must be quick, accurate and effective. Since people can not go near or into the scene directly after the accident to collect information or monitor the condition, the rescue work may be delayed. How to obtain macroscopic information of the accident timely, the detailed information correctly and control and monitor the whole situation dynamically, especially under special environmental conditions become very important. Meanwhile, based on the scale, nature, characteristics and site environment of the accident, how to obtain the parameter information in time is an urgent problem to be solved in China's emergency rescue system.

At present, emergency rescue equipment in many safety production and emergency command centers are imperfect, especially the lacking of data acquisition equipment and additional tools in accident scenes. However, Mini-UAVs loaded with emergency rescue equipment can take off from small platform and with its unique features like low speed, stability and hovering; it can carry out point detection and tracking comprehensively at some high places or narrow spaces where emergency rescue crew cannot reach.

\section{Mini-UAV(UNMANNED AERIAL VEHICLE) AND REMOTE SENSING DETECTION SYSTEM}

The UVA is an unmanned aircraft which has its own power system and can be monitored, loaded with several equipments, used to perform many tasks and recycled. The combination of UAVs and remote sensing technique, also named UAV Remote Sensing is to adopt the advanced unmanned aerial vehicle technology, remote sensing sensor technology, remote telemetry technology, communication technology, GPS differential positioning technology and remote sensing technique to make UAVs automatic, intelligent and specific in rapidly accessing to remote information of territory, resources and environment and then to carry on the work of remote sensing data processing, modeling, and analyzing of application.. It has many advantages like low-cost, low-loss , low-risk and can be recycled. The application has been expended from military fields (detecting, early warning .etc) to non-military fields like resource exploration, meteorological observations and handling of emergency. The traditional satellites are inferior to UAV Remote Sensing which is more effective and has a higher resolution[1].

\section{A. Mini-UAV}

The unmanned aerial vehicle whose wingspan is usually between 100 to $300 \mathrm{~cm}$ and the weight of which is often less than 50 kilograms is called Mini-UAV while that with 15 to $100 \mathrm{~cm}$ 's long wingspan and weighs less than one kilogram is often called Micro-UAV . However, there is no standard rule to classify them regarding to the size. Mini-UAV not only has the advantages of UAVs, but also it is widely used because of its small size and flexibility [2,3]. The Mini-UAV whose wingspan, the length of the plan and diameter are all between 100 to $300 \mathrm{~cm}$ and the weight less than 50 kilograms is used in emergency rescue of major accidents of hazardous chemicals 。

\section{B. The formation and working procedures of UAV remote sensing monitoring system}

UAV remote sensing monitoring system is the third generation of remote sensing platform after traditional aviation, 
aerospace remote sensing platform. It can access to the remote sensing information of geography, resources and environment and then continue the work of collecting, proceeding and analyzing of remote sensing data quickly. Since this system is power-driven, economic and responds quickly, it now has been the hot topic of researches all around the world. Nowadays, it has gradually developed from theoretical approaches to field applications, becoming one of the major aviation remote sensing technologies [4]. The application of UAV remote sensing monitoring system to emergency rescue of accidents of dangerous chemicals can effectively improve the accuracy, reliability and timeliness of the basic data for rescue, which provides important technique support for rescue work and lays a good foundation for the security department's accurate, reasonable and efficient decision-making.

UAV remote sensing monitoring system is mainly composed of eight parts: UAV flight platform, data link, remote sensing monitoring platform, real-time monitoring platform, ground-based monitoring system, ground support system (including the launch system), remote sensing and detection systems and post-processing system. As shown in Fig. 1 Mini-UAV equipment system[5].

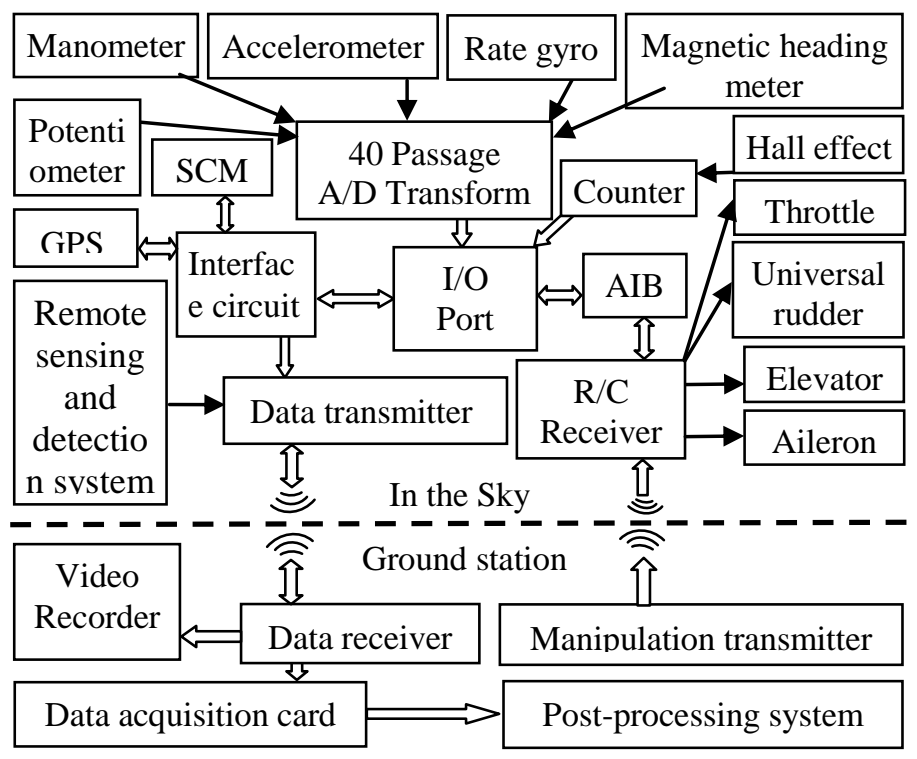

Fig. 1. Mini-UAV equipment system

The working procedures of Mini-UAV remote sensing monitoring system are composed of three parts: the early stage office operation, outside operation and late stage operation. The advanced domestic UAV technology is to take-off by the manual remote control. After the UAV enters the routes, it will be cut into the automatic flight status. The inertial navigation platform and Global Positioning System will then be used to control the UAV to fly in the predetermined routs and timely send the aircraft's flight data to the ground. Once remote sensing tasks were finished, the flight status would be switched to manual remote control. The late stage operation includes downloading and organizing of remote sensing data; data preprocessing, data interpretation and results formation, etc. The detailed procedures of the late stage operation may be different because of the different working purposes or needs of data[6].

\section{MAJOR ACCIDENTS OF DANGEROUS CHEMICALS AND THE CHARACTERISTICS OF THE RESCUE}

Major accidents of dangerous chemicals include fire, explosion, poisoning, etc. These accidents have the following characteristics. First, unexpected abruptness with large quantity of poison leakage. Second, a wide range of toxicity. After the accidents, the polluted gas quickly spreads to the downwind places and toxic and hazardous substances can seriously pollute the air, ground, road, and production and living facilities, hazards range within a short period of time can be up to tens or even hundreds of square kilometers. Third, various forms of damage. It does great damage to people and the environment. For example, toxic damage, burns, mechanical damage of the explosion, etc.

The emergence rescue of accidents of hazardous chemicals is different from the normal rescue of accidents or detection of the environment. It has several characteristics as follow. First, people can not reach the position because of the leakage of hazardous chemicals, fire, and the high temperature after the explosion, the polluted gas or the polluted earth so that the latest information can not be collected. Second, the bad influence may be extensive so that the overall situation can not be grasped. As can be illustrated by the leakage of chlorine, which can be very poisonous and spreads by the wind quickly. Third, the high risk of fire explosion. It's too risky for people to get into the scene of the series explosion of multi-tank and reactor.

\section{THE APPLICATION OF MINI-UAV IN EMERGENCY RESCUE OF ACCIDENTS OF HAZARDOUS CHEMICALS.}

According to the characteristics of accidents of hazardous chemicals, the passage gives a brief introduction of application of Mini-UAV in emergency rescue of major leakage of toxic and harmful gases, fire and explosion of hazardous chemicals, leakage of toxic and hazardous liquid material.

\section{A. The emergency rescue of accidents of leakage of toxic and harmful gases}

After major leakage occurs, toxic and harmful gases by wind can quickly spread to a few kilometers away, which results in mass poisoning. So while controlling the source of the leakage, the rescue members should quickly evacuate residents within a certain range of the leak points under the wind direction and rescue the people who have already been affected. In this series of emergency tasks, the key information like warning and the evacuation range, retreating routs and the allocation of rescue materials should be confirmed by the fast, accurate, and effective on-site data feedback.

The usages of Mini-UAV in such accidents are as follow. First, Mini-UAV can be equipped with a mobile automatic air monitoring platform so as to monitor the atmosphere of the target area and keep abreast of the concentration of toxic and hazardous substances of the key points. After that, the division of the warning area and evacuating routes can be formulated. 
Second, when the pollution factor cannot be monitored by automatic monitoring platform, we can equip the sampler to acquire the atmospheric samples and send them back to the lab for detection. Third, the safe operation of UAV remote sensing monitoring system can be guaranteed so that it can go into the high-risky places to carry out the tasks. Thus major risks can be avoided. Fourth, when the toxic and harmful gases spread, Mini-UAV can collect information quickly from the sky about downwind toxic and harmful gas diffusion situation and affected regions' geographical landscape, social environment, and to provide timely and effective data reference for the rescue.

\section{B. Emergency rescue of fire and explosion of dangerous chemicals}

Dangerous chemicals can easily lead to a chain reaction of explosions in the fire, causing casualties and significant economic and property losses. Burning rapidly and strong thermal radiation threat to the firefighters and other security devices and equipment. Fire and explosion of dangerous chemicals include fire source type (resulted by burning or leaking), regenerative type (resulted by natural causes or accidents of experiment) and latent type (caused by heat transfer or destroying of balance). The six fire explosion types are all related to heat. Accurate collection of regenerator and temperature in various dangerous fire and explosion accident scenes is of key importance to the formulation of emergency rescue and the prevention of the accident caused by the former one.

The usages of Mini-UAV in these kinds of accidents are listed in the following part. First, Mini-UAV can be equipped with infrared temperature sensing device to detect the temperature of the surroundings of the accident scene, the unexploded reactor in the line, intermediate storage tanks and pipelines so as to prevent the following accidents caused by the former one. Second, Mini-UAV can get into the areas where vehicles, equipment and rescue personnel should not enter to collect information. Third, Mini-UAV can be equipped with life detectors, auxiliary personnel rescue.

\section{Emergency rescue of toxic and hazardous liquid material spill}

Major spill of toxic and hazardous liquid contains two major types .First, toxic and hazardous liquid directly leaks and gas leaks. Second, the wastewater used in putting out fire in the fire and explosion pollutes a large number of natural water body. Traditional water sample testing usually test the water from a certain place of the water body to evaluate the whole situation of the water body, which of course has its limitations. UAV remote sensing detection system has the features of wide horizon, timely and continuous working so that it can quickly identify the state of the environment. With the equipped multispectral imager generating multispectral images, we can have a comprehensive monitoring of surface water quality of the environment and get a whole picture of the water about the eutrophication, blooms, water clarity, suspended solids, sewage outfall pollution situation. Mini-UAV equipped with lowaltitude controlled spray equipment carry out the decontamination work while dispersant spraying operations are an important means of oil spill treatment.

\section{APPLICATION EXAMPLES OF MINI-UAV IN THE HANDLING OF OIL SPILL EMERGENCY}

July 16th, 2012, 6:00 p.m., the pipelines in Xingang, Dalian exploded. CAS Shenyang Institute of Automation sent the rotor UAV to relevant areas in Dalian on July 20th. carry out the work of oil spill monitoring and disposal of the situation. Rotor UAV equipped with oil spill detection load and low-altitude controlled spraying equipment finished the low-flying detection and certain work above the polluted area and provided the data of oil spills and assisted the relevant departments to effectively remove oil spills and minimized losses. During the work, the rotor UAV overcame the difficulties of satellite remote sensing resolution and the problem that the normal plan flied by man can not fly at night or in bad weather. It not only carry out the timely detection work in good weather, but also worked at rainy night and foggy day with visibility less than two meters. It used infrared and other spectral information to have 24 hours' monitoring of the oil spill, and had achieved good results.

Dispersant spraying operations is an important means of the processing of the oil spill; however, the excessive spraying or insufficient spraying will bring adverse consequences like environmental pollution and the invalid work. The volume used in rotor UAV low-altitude spraying operations can be based on previous oil spill monitoring data. What's more it can also help to achieve accurate control of the sprayed areas to maximize efficiency and reduce environmental pollution.

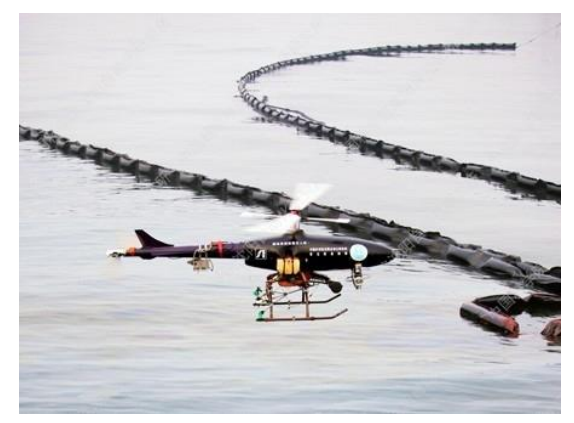

Fig. 2. Rotor UAV in Oil Spill Emergency Treatment

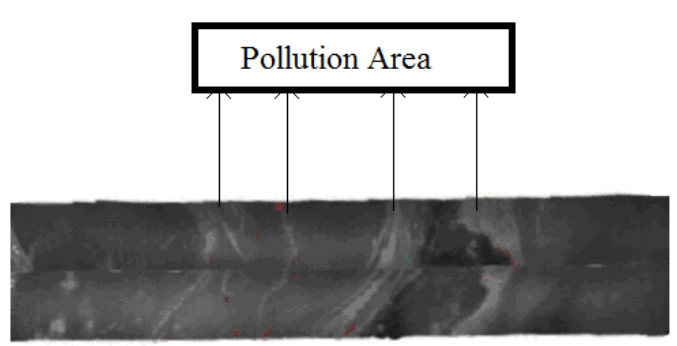

Fig. 3. The results of the detection (From the Chinese Academy of Sciences, Institute of Remote Sensing Applications) 


\section{THE PROSPECTS OF MINI-UAV IN EMERGENCY RESCUE OF MAJOR ACCIDENTS OF DANGEROUS CHEMICALS}

The rescue of major accidents of dangerous chemicals should be quick, accurate and effective. Mini-UAV have a broad prospect in the emergency rescue. The following passage will discuss the advantages and disadvantages as well as the methods for improvement of the Mini-UAV.

\section{A. The advantages of Mini-UAV in emergency rescue of major accidents of dangerous chemicals.}

Mini-UAV not only satisfy the needs for quick response, accuracy and effectiveness, but also they have the following advantages: 1) the capability of rapid emergency response. UAV remote sensing system has small size, light weight and its takeoff and landing are less restricted by the venue so that it can respond quickly ; 2) Mini-UAV are equipped with a variety of data acquisition machines so that they can have direct and accurate access to the on-site first-hand information. Mini-UAV can be equipped with a sense of sophisticated detection equipment, including digital cameras, infrared temperature sensing devices, synthetic aperture radar, automatic air monitoring platform. Choices of the acquisition are determined by the rescue mission. People can select different types of acquisition based on a rescue mission to obtain the corresponding type rescue data; 3) the Mini-UAV' size are moderate, which enables them adapt to the work in various regions of a major emergency rescue site; 4) the security of the job can be ensured. UAV remote sensing system uses autonomous and ground control practices to make it fly to the high-risk areas to work, and avoid the risk of the safety of the flight crew and ground staff; 5) controlled spraying device can be equipped to carry out low-altitude decontamination work in polluted environment; 6) Mini-UAV are capable of performing the tasks under the "3D" environment. The socalled 3D, Dull, Dirty and Dangerous environment. These also often appear in a major accident environment; 7) Mini-UAV are able to obtain data under the cloud. UAVs can fly under the cloud which makes up for the shortcoming of satellite optical remote sensing which has a defect that it can not get the image in cloudy weather; 8)remote sensing data acquisition is much cheaper. Acquisition of UAV remote sensing system and the operating costs are significantly lower than those of the satellite and manned aircraft. The technical requirements of the venue and the staff is lower than those of manned aircraft, and the routine maintenance is comparatively easy which reduce the cost of remote sensing data acquisition.

\section{B. The disadvantage and its improvement of Mini-UAV in emergency rescue of hazardous chemicals}

At present, the disadvantages of Mini-UAV include: 1) in major accidents of hazardous chemicals, there are flammable and explosive gases, but small unmanned aerial vehicles often do not have the explosion-proof performance; 2)in the fire explosion scene, airflow changes quickly, but Mini-UAV weigh light, resulting in poor stability, and deformation of aerial photographs; 3 ) when design the routs, people cannot get access to many available flight points. In addition, airborne GPS positioning error and yaw factors are unavoidable, resulting in relatively small effective shooting area for each flight; 4) Mini-UAV can not use professional aerial camera, attitude measuring instrument, stable platform and other auxiliary equipment, the relevant parameters of the resulting image are not enough, leading to the difficulties of the postimage processing and image accuracy assessing; 5) 3D video output can not be achieved at the fire scene of the explosion ; 6) the detection data of a wide range of toxic and hazardous gas needs to be developed

The methods to solve the above problems are to be discussed next. The explosion-proof design should be used in Mini-UAV, and the balance, stability and control of the aircraft should be improved. It's necessary to design different types of mini UAVs to perform different tasks. On the basis of the existing GPS navigation system, GPS / Inertial navigation / combination of image matching navigation methods should be combined to improve the accuracy of navigation. Structure digital platform based on the three-dimensional remote sensing. Develop and use three-dimensional landscape modeling software to transfer remote sensing images into threedimensional visualization products. It's of great necessity to research on and develop the intelligent recognition technology of remote sensing information, equip the UAVs with intelligent sensors to realize real-time monitoring of multi-class pollution factor, and develop various software for the late stage operation The Mini-UAV can use existing geographic information to complete image stitching, correction and interpreting work in a short period of time according to the needs of different users.

\section{ACKNOWLEDGMENT}

This work was supported in part by the National Natural Science Foundation of China under Grant 10903003, and the Construction Plan of science and technology infrastructure for Jiangsu under Grant BM20122067.

\section{REFERENCES}

[1] Jin Wei, Ge Lihong, Du Huaqianag. The Development of UAV Remote Sensing and Its Application. [J]. Remote Sensing Information, 2009 (1): 88-92

[2] Xu Wen. The Development Status of Small and Micro UAVs and The Equipment. [J]. Cruise Missiles, 2003 (11): 20-25

[3] Cui Xiuming, Wang Weijun, Fang Zhenping. The Development Status of Mini-UAV and The Analysis of The Problems. [J]. Flight Mechanics, 2005, 23(1): 14-18

[4] Lei Tianjie, Li Changchun, He Xiaorong. The Application of UAV Aerial Remote Sensing Detection System in Emergency Rescue. [J]. Journal of Natural Disasters, 2011,20(1): 178-183

[5] Ella M Atkins Solus: An Autonomous Aircraft for Flight Control and Trajectory Planning Research [R].Proceeding of the American Control Conference Philadelphia, Pennsylvania, 1998.

[6] Zhu Jinghai, Xu Guang, Liu Jiabin. Research of The Application of UAV Remote Sensing Monitoring System in the Field of Environmental Protection. [J]. Environmental Protection and Recycling Economy, 2011, (9): 45-48 